\title{
Patients' opinions on enhanced recovery after surgery perioperative care principles: a questionnaire study
}

\author{
Anna Zychowicz ${ }^{1}$, Magdalena Pisarska ${ }^{1,2}$, Anna Łaskawska ${ }^{1}$, Monika Czyż ${ }^{1}$, Jan Witowski ${ }^{1,2}$, Michał Kisielewski ${ }^{1,2}$, \\ Jan Kulawik ${ }^{1}$, Andrzej Budzyński ${ }^{1,2}$, Michał Pędziwiatr ${ }^{1,2}$ \\ ${ }^{1} 2^{\text {nd }}$ Department of General Surgery, Jagiellonian University Medical College, Krakow, Poland \\ ${ }^{2}$ Centre for Research, Training and Innovation in Surgery (CERTAIN Surgery), Krakow, Poland
}

Videosurgery Miniinv 2019; 14 (1): 27-37 DOI: https://doi.org/10.5114/wiitm.2018.77261

\begin{abstract}
Introduction: The enhanced recovery after surgery (ERAS) protocol relies on patients' compliance and their awareness of its principles. Patients' views on ERAS implementation have never been evaluated in Poland before.

Aim: To analyse patients' opinions about this protocol.

Material and methods: One hundred forty consecutive patients undergoing elective laparoscopic procedures in which ERAS was implemented were asked to join the study. Out of them, 120 fulfilled the trial criteria and were surveyed once before and twice after surgery. A 22-question survey about the patient's perception of ERAS principles was presented upon admission and on the day of discharge. A telephone follow-up was performed 2 weeks after discharge. Results: Patients reported the need for being counselled by the anaesthetist/surgeon as the most important element of the protocol. Items such as being free of pain, being free of gastrointestinal symptoms, and being free of catheter were rated more highly preoperatively than on the day of discharge, whereas telephone check-up call, being able to drink and eat early after surgery, avoiding bowel preparation, avoiding preoperative fasting and reducing postoperative intravenous fluids were rated more highly on the day of discharge. Pain was still present in over half of patients two weeks after discharge. Only $4.76 \%$ of patients admitted in the telephone survey that they were afraid of early discharge. Shortening of the length of hospital stay thanks to ERAS was widely appreciated among all patients who did not find it necessary to spend a longer time in the hospital.

Conclusions: Polish patients approve the ERAS protocol as modern perioperative care. Patients emphasize the need for preoperative counselling and painless recovery.
\end{abstract}

Key words: fast-track surgery, perioperative care, enhanced recovery after surgery, patients' opinions.

\section{Introduction}

There is growing evidence that the introduction of Enhanced Recovery After Surgery (ERAS) protocols into daily clinical practice may have a positive impact on postoperative outcomes in abdominal surgery. Encouraging results from colorectal surgery resulted in the introduction of ERAS to other surgical disciplines. Briefly, ERAS is a structured perioperative protocol that includes pre-, intra- and postoperative interventions that when combined are thought to significantly reduce surgical trauma, thus leading to faster postoperative recovery [1]. Thanks to ERAS it is possible to reduce overall morbidity, shorten length of hospital stay (LOS) and reduce treatment costs [2-4]. Although the number of hospitals implementing new perioperative care is rapidly growing, the safety of ERAS implementation is still a matter 
of dispute. It is particularly pronounced in Poland, as shown by Kisielewski et al. [5]. Although there is strong evidence supporting ERAS, most of its elements are consistently denied by surgeons, who in most cases believe in unproven dogmas [6]. This results in difficulties in changing perioperative care and poor compliance with the ERAS protocol [7].

Moreover, ERAS implementation requires full understanding not only from the medical staff but also patients involved in the recovery process [8]. Unlike in the traditional clinical pathway, ERAS strongly relies on full patient cooperation and understanding the principles of modern care, rapid mobilization and early discharge [9]. Therefore, it is crucial to get to know patients' attitudes to modern perioperative care. However, their opinion has never been investigated in our country.

\section{Aim}

The aim of the study was to explore patients' views on ERAS in order to establish what patients' priorities are in terms of perioperative care.

\section{Material and methods}

\section{Patients and questionnaires}

The study included all consecutive patients scheduled for elective laparoscopic colorectal, gastric, pancreatic, hepatic and bariatric procedures between October 2014 and March 2016. They were all asked to complete a questionnaire during hospital stay (before surgery and on the day of discharge). Patients who did not give consent to participate or developed major complications (defined as Clavien-Dindo grade III or higher) as well as those who did not answer all questions were excluded from the analysis.

A questionnaire was created according to a modified survey used by Hughes et al. [9]. Initially questions were formulated in English and then translated into Polish. They aimed to quantify the patients' perception of the importance of individual enhanced recovery items. The original questionnaire was validated by its authors.

The questionnaire is presented in Supplementary material.

The survey was divided into two parts. The first part incorporated the major domains of ERAS principles such as pain control, gut restitution, mobility, overall function and hospital discharge. Patients were asked to complete the survey comprising
22 questions on admission (the day before surgery) and on the day of discharge from hospital (the same 22 questions and 7 additional questions regarding postoperative symptoms). It was followed by a verbal explanation of the study goals and instructions how to fill in the questionnaire. Questions were related to ERAS items and each of them featured an 11-point Likert scale (0-10). Patients were asked to score the importance of each particular ERAS item. The scale used indicator statements of "not important" and "very important" at the relevant extremes of the scale to assist with scoring. On the day of discharge additional questions related to postoperative symptoms were asked.

In addition to the main questionnaire patients were contacted via telephone 2 weeks after discharge and were asked 9 additional questions (the second part of the survey). They contained questions regarding symptoms that persisted after leaving the hospital as well as the general impression of the ERAS protocol, its usefulness and acceptance. There was a separate question whether, in their opinion, the hospital stay was long enough.

All procedures performed in studies involving human participants were in accordance with the ethical standards of the institutional and national research committee and with the 1964 Helsinki Declaration and its later amendments or comparable ethical standards.

\section{Statistical analysis}

It was previously calculated that at least 105 patients are needed in order to reach the minimal sample size as described by Rosner [10]. All data were analysed with StatSoft Statistica 13. The results are presented as mean \pm standard deviation (SD), median and interquartile range (IQR). The study of categorical variables used the $\chi^{2}$ test of independence. The Shapiro-Wilk test was used to check for normal distribution of data and Student's $t$ test was used for normally distributed quantitative data. For non-normally distributed quantitative variables, the Mann-Whitney $U$ test was used. For dependent variables the Friedman test was used. Results were considered statistically significant when the $p$-value was less than 0.05 .

\section{Results}

Initially, 140 patients were included in the analysis (preoperative questionnaire). After excluding 
those with major complications (16 patients) and 4 patients who did not respond to either the discharge questionnaire or telephone call, 120 individuals were analysed. Their demographic characteristics are presented in Table I.

Figure 1 shows overall scores for 22 questions that were asked preoperatively and on the day of discharge. In answers to 9 questions the difference was statistically significant. Items such as being free of pain, being free of gastrointestinal symptoms, and being free of a catheter were rated higher preoperatively than on the day of discharge, whereas telephone check-up call, being able to drink and eat early after surgery, avoiding bowel preparation, avoiding preoperative fasting and reducing postoperative intravenous fluids were rated higher on the day of discharge. In the remaining 13 questions, there were no differences in pre- and postoperative score.

When analysing postoperative symptoms, we observed that pain was the main issue. However, a majority of patients complained of fatigue, eating and drinking problems as well as gastrointestinal tract symptoms (bloating, constipation or diarrhoea). A relatively small proportion of patients had a problem with quick mobilization (Figure 2).

A telephone questionnaire was conducted 2 weeks after discharge (Figure 3). Pain and fatigue were still present in more than half $(57.14 \%$ and $56 \%$, respectively) of patients, whereas gastrointestinal symptoms did not subside in one third (34.52\%) of them. Moreover, less than $10 \%$ required medical assistance after discharge. One in ten patients admitted they were afraid of early discharge. However, more than $95 \%$ did not find a longer hospital stay necessary. All patients fully approved and recommended the ERAS protocol as routine perioperative care.

\section{Discussion}

This study is the first to show patients' full acceptance of changes in the perioperative care thanks to the ERAS protocol in our country. We have found that their expectations are coherent with the ERAS philosophy. In addition, we have observed that although a large proportion of patients present minor symptoms after discharge, practically all of them did not find the need to stay longer in the hospital.

The principles of Enhanced Recovery After Surgery (formerly fast-track surgery) were formulated more than 20 years ago by Professor Kehlet [1]. Sub-
Table I. Demographic characteristics of patients $(n=120)$

\begin{tabular}{|c|c|c|}
\hline \multirow[t]{2}{*}{ Parameter } & \multicolumn{2}{|c|}{ Value } \\
\hline & $N$ & $\%$ \\
\hline \multicolumn{3}{|l|}{ Sex: } \\
\hline Female & 62 & 51.7 \\
\hline Male & 58 & 48.3 \\
\hline Mean age [years] & \multicolumn{2}{|c|}{$54.0 \pm 15.7$} \\
\hline \multicolumn{3}{|l|}{ ASA grade: } \\
\hline ASA 1 & 4 & 3.3 \\
\hline ASA 2 & 83 & 69.2 \\
\hline ASA 3 & 33 & 27.5 \\
\hline \multicolumn{3}{|l|}{ Comorbidities: } \\
\hline Any comorbidity & 90 & 75 \\
\hline Cardiovascular & 21 & 17.5 \\
\hline Hypertension & 69 & 57.5 \\
\hline Diabetes & 33 & 27.5 \\
\hline Pulmonary & 16 & 13.3 \\
\hline Renal & 3 & 2.5 \\
\hline Liver & 35 & 29.2 \\
\hline \multicolumn{3}{|l|}{ Type of surgery: } \\
\hline Bariatric & 51 & 43.5 \\
\hline Colon/rectum & 38 & 32.7 \\
\hline Gastric & 16 & 13.3 \\
\hline Pancreatic & 13 & 10.8 \\
\hline Liver & 2 & 1.7 \\
\hline
\end{tabular}

sequently, it has become evident that ERAS may be beneficial in many surgical disciplines and has been widely studied in colorectal, gastric, pancreatic, liver and bariatric surgery $[4,11-15]$. ERAS is associated with reduced surgical trauma leading to diminished postoperative catabolism, thus accelerating postoperative functional recovery [16]. In addition to shortened LOS, thanks to ERAS a reduced complication rate can be observed. Previous studies have clearly confirmed the safety of modern perioperative care which despite shorter hospital observation is not associated with an increased number of readmissions $[2,4,17,18]$. Despite existing evidence, the adop- 
To be counselled preoperatively by the anaesthetist or surgeon

To be completely free of pain*

To be operated with the use of minimally invasive techniques

To be able to move independently shortly after the procedure

To get painkillers immediately when needed

To be completely free of nausea and vomiting

To be able to return quickly to everyday activities

To be able to move the bowels as soon as possible (no constipation and...)

To be checked up by telephone after hospital discharge*

To be catheterized as short as possible*

To be allowed normal diet at will after the operation

To be warmed up in the operating room during surgery

To avoid the use of nasogarstic tube

To avoid the use of abdominal drains

To be discharged from the hospital as quickly as possible

To avoid sedative drugs before surgery

To be able to drink and eat as soon as possible after surgery

To avoid bowel cleaning when possible

To be able to drink shortly before the surgery

To be able to reduce the amount of intravenous fluids postoperatively

To avoid fasting before surgery

$\square$ Before surgery

$\square$ On the day of discharge

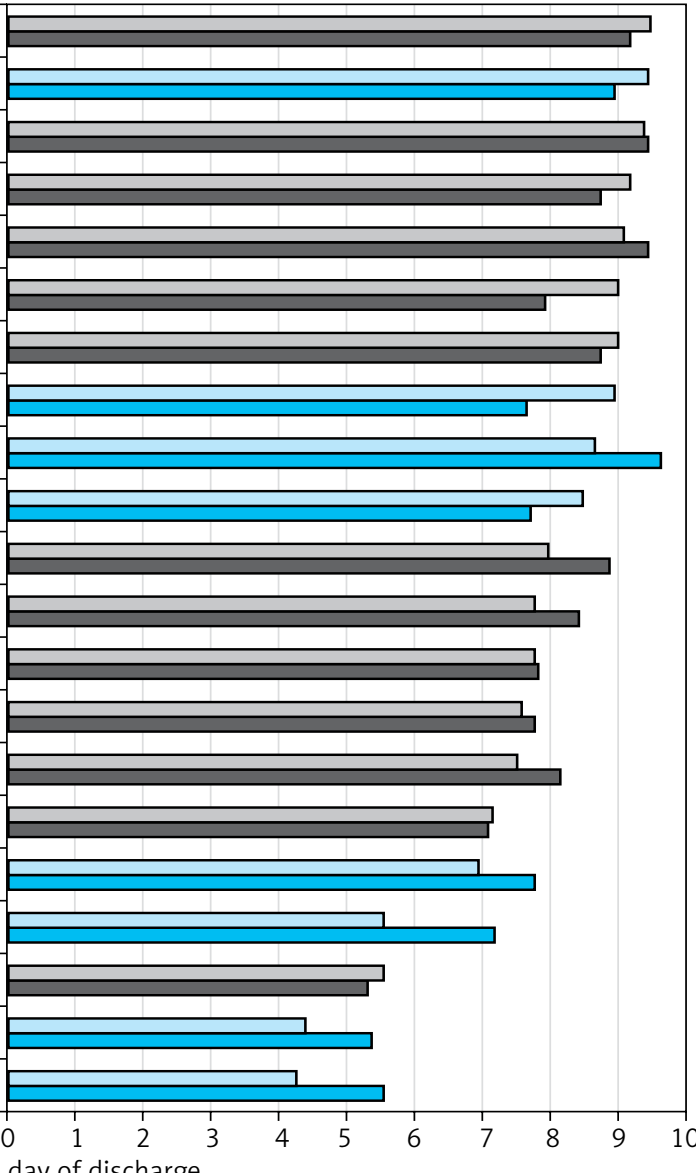

Figure 1. Patients ratings of ERAS items importance and goals. Answers to questions marked with asterisk $\left(^{*}\right)$ and blue-coloured bars were statistically significant. Questions are listed in order of given score, descending

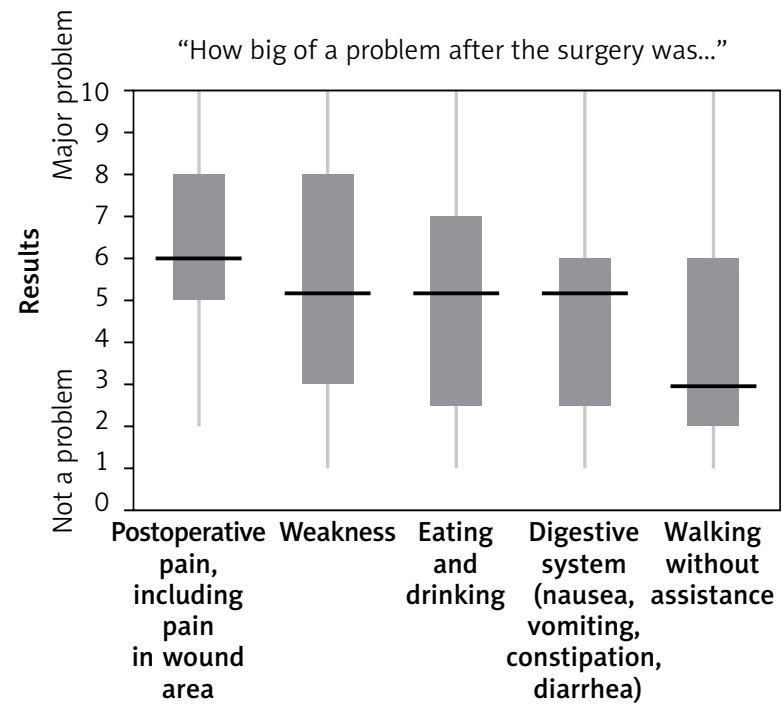

Figure 2. Rating of postoperative symptoms and difficulties in the group of patients on the day of discharge tion of ERAS remains slow, and therefore deviation from the protocol is often observed [19].

The response rate to all questions and steps of the questionnaire among patients included in the study was $85.7 \%$. This is relatively high taking into consideration telephone calls 2 weeks after discharge and exclusion of those developing complications. The results of the questionnaire clearly show that in general patients found ERAS items to be similar to their expectations. Interestingly, the highest score (> 9.4 points) was observed for full patient counselling and full information on the treatment plan together with preoperative meeting with the surgeon and anaesthetist (rated higher than being free of pain). ERAS guidelines clearly state that detailed information given to patients before the procedure about surgical and anaesthetic procedures (ideally by those involved in the surgical procedure) 


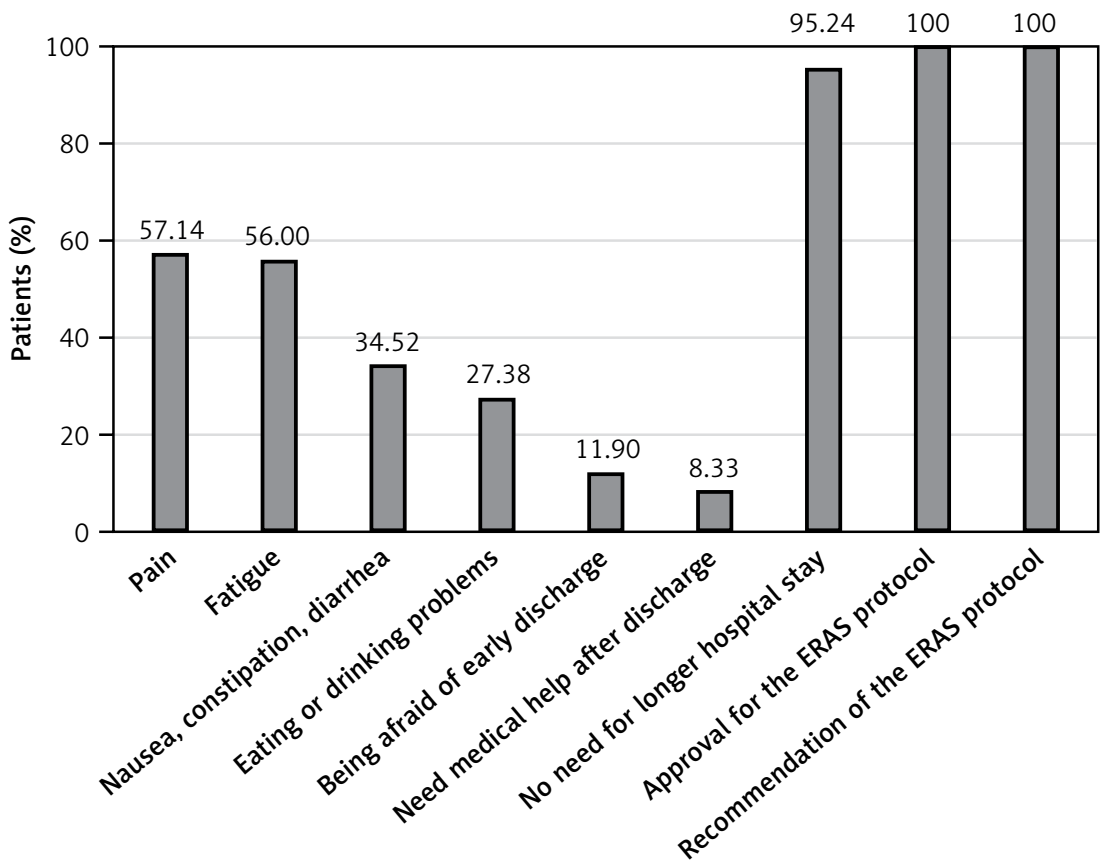

Figure 3. Patients' response to questions asked via telephone 2 weeks after discharge

may diminish fear and anxiety and enhance postoperative recovery and quicken hospital discharge [20-24]. Given the results of this questionnaire, one has to bear in mind that full and comprehensive explanation of the treatment plan is of great value not only for the medical staff but for the patients as well.

Patients paid more attention to items directly influencing their postoperative condition and physical feelings: being free of pain, no nausea and vomiting, early mobilization and return to daily activities. All adverse symptoms are strongly related to their postoperative well-being and understanding of appropriate recovery from surgical trauma. Therefore, maximum attention should be paid in order to prevent the occurrence of unpleasant symptoms by prevention of pain, postoperative nausea and vomiting, removal of catheters and drips as well as early ambulation.

We observed that items that were strongly correlated with shortened LOS in previous studies (appropriate fluid management, early postoperative oral feeding, no use of drains and nasogastric tubes and omission of bowel preparation) were scored lowest by our patients $[12,14]$. They were not as important for them as they are for clinicians. On one hand, this may be due to patients' partial understanding of the pathophysiology of surgical trauma. On the other, it has to be noted that this might be the result of the study group characteristics. Nasogastric tubes are not used in any of our patient, we adopted no drain policy for most of our procedures and bowel preparation is used only in selected cases. At first, these items may seem unnecessary for all those who did not experience their presence and unpleasantness. However, the situation changes on the day of discharge. We noticed significant changes in pre- and postoperative scoring of these particular items. Although being free of pain, no nausea and vomiting, no constipation and bloating, and shorter catheterisation were rated lower postoperatively, telephone follow-up after discharge, early enteral feeding, no bowel preparation, no preoperative fasting and intravenous fluid restriction were rated higher after than before surgery. In our opinion, this clearly shows that patients realize the importance of items leading to functional recovery and being independent of medical staff only after being discharged home.

Perhaps even more interesting was the observation of the second part of the study related to persistent postoperative symptoms on the day of discharge. As shown in Figure 2, in a large proportion of patients the functional recovery is not full on the day of discharge. Pain and fatigue seem to be the most problematic, but there are also problems with food and fluid consumption and bowel function in general. This raises the question whether the discharge 
comes too early. However, analysis of the telephone call 2 weeks after surgery shows that pain and fatigue are still present in almost $50 \%$ of cases and bowel function is not recovered in one third of them. It obviously does not mean patients' hospital stay should be prolonged. As shown in the results, more than $95 \%$ of patients expressed their satisfaction with the LOS and 100\% approved and recommended the ERAS concept. This important finding, together with the data on the unchanged readmission rate after introduction of ERAS in comparison to traditional care, should clearly confirm that when patients' discharge criteria are met, patients are ready to go home. As shown by King et al., it usually takes months until the patient fully recovers after laparoscopic colorectal resection [25]. Therefore, they desire post-discharge follow-up and close contact with their treating medical unit also after leaving medical institutions. We think that whereas preoperative counselling is extremely important in calming down patients, the post-discharge follow-up protocol plays a crucial role after going home. Only in such an environment do patients feel safe and are able to accept ERAS principles and early discharge.

Our study has certain limitations. The study sample (although calculated and correctly powered) is still small taking into consideration the heterogeneous study group. Including patients undergoing various types of surgery may introduce bias. However, the aim of ERAS is to provide a similar type of perioperative care regardless of the procedure. Therefore, in our opinion, an analysis of consecutive patients undergoing various types of surgery speaks for the applicability of the study. Most of our patients underwent a laparoscopic procedure which might have affected their answer to particular survey questions. Perhaps those undergoing an open procedure would have different opinions. Due to the small sample size, we were not able to compare patients after open laparotomy with laparoscopic cases as well as groups undergoing different procedures. In addition, we did not evaluate differences in opinions of patients who had been operated on before and those having the first surgery - a previous operation certainly might have influenced their attitudes. And finally, although some of the differences are statistically significant, they may be considered of dubious clinical significance (e.g. 1 point on an 11-point scale). Therefore, our results should be interpreted with caution.

\section{Conclusions}

Our study confirmed that Polish patients approve the ERAS protocol as modern perioperative care. Patients emphasize the need for preoperative counselling and fast and painless recovery. Shortening of the length of hospital stay thanks to ERAS was widely appreciated among all patients who did not find it necessary to spend a longer time in the hospital.

\section{Conflict of interest}

The authors declare no conflict of interest.

\section{References}

1. Kehlet H, Wilmore DW. Evidence-based surgical care and the evolution of fast-track surgery. Ann Surg 2008; 248: 189-98.

2. Greco M, Capretti G, Beretta L, et al. Enhanced recovery program in colorectal surgery: a meta-analysis of randomized controlled trials. World J Surg 2013; 38: 1531-41.

3. Pędziwiatr $M$, Wierdak $M$, Nowakowski $M$, et al. Cost minimization analysis of laparoscopic surgery for colorectal cancer within the enhanced recovery after surgery (ERAS) protocol: a single-centre, case-matched study. Videosurgery Miniinv 2016; 11: 14-21.

4. Małczak P, Pisarska M, Major P, et al. Enhanced recovery after bariatric surgery: systematic review and meta-analysis. Obes Surg 2017; 27: 226-35.

5. Kisielewski $M$, Rubinkiewicz $M$, Pędziwiatr $M$, et al. Are we ready for the ERAS protocol in colorectal surgery? Videosurgery Miniinv 2017; 12: 7-12.

6. Lassen K, Hannemann P, Ljungqvist O, et al. Patterns in current perioperative practice: survey of colorectal surgeons in five northern European countries. BMJ 2005; 330: 1420-1.

7. Maessen J, Dejong CHC, Hausel J, et al. A protocol is not enough to implement an enhanced recovery programme for colorectal resection. Br J Surg 2007; 94: 224-31.

8. Grol R, Grimshaw J. From best evidence to best practice: effective implementation of change in patients' care. Lancet 2003; 362: $1225-30$.

9. Hughes M, Coolsen MME, Aahlin EK, et al. Attitudes of patients and care providers to enhanced recovery after surgery programs after major abdominal surgery. J Surg Res 2015; 193 : 102-10.

10. Rosner B. Fundamentals of biostatistics. Nelson Education, 2015.

11. Kehlet H, Wilmore DW. Multimodal strategies to improve surgical outcome. Am J Surg 2002; 183: 630-41.

12. ERAS Compliance Group. The impact of enhanced recovery protocol compliance on elective colorectal cancer resection: results from an international registry. Ann Surg 2015; 261: 1153-9.

13. Kisialeuski M, Pędziwiatr M, Matłok $M$, et al. Enhanced recovery after colorectal surgery in elderly patients. Videosurgery Miniinv 2015; 10: 30-6. 
14. Pędziwiatr M, Pisarska M, Kisielewski M, et al. Is ERAS in laparoscopic surgery for colorectal cancer changing risk factors for delayed recovery? Med Oncol 2016; 33: 25.

15. Pędziwiatr M, Matłok M, Kisialeuski M, et al. Short hospital stays after laparoscopic gastric surgery under an enhanced recovery after surgery (ERAS) pathway: experience at a single center. Eur Surg 2014; 46: 128-32.

16. Ljungqvist O. Jonathan E. Rhoads lecture 2011: Insulin resistance and enhanced recovery after surgery. JPEN J Parenter Enteral Nutr 2012; 36: 389-98.

17. Pisarska M, Małczak P, Major P, et al. Enhanced recovery after surgery protocol in oesophageal cancer surgery: systematic review and meta-analysis. PLoS One 2017; 12: e0174382.

18. Pędziwiatr M, Pisarska M, Kisielewski M, et al. ERAS protocol in laparoscopic surgery for colonic versus rectal carcinoma: are there differences in short-term outcomes? Med Oncol 2016; 33: 56.

19. Smart NJ, White P, Allison AS, et al. Deviation and failure of enhanced recovery after surgery following laparoscopic colorectal surgery: early prediction model. Colorectal Dis 2012; 14: e727-34.

20. Egbert LD, Battit GE, Welch CE, Bartlett MK. Reduction of postoperative pain by encouragement and instruction of patients. A study of doctor-patient rapport. N Engl J Med 1964; 270: 825-7.

21. Kiecolt-Glaser JK, Page GG, Marucha PT, et al. Psychological influences on surgical recovery. Perspectives from psychoneuroimmunology. Am Psychol 1998; 53: 1209-18.

22. Gustafsson UO, Scott MJ, Schwenk W, et al. Guidelines for perioperative care in elective colonic surgery: enhanced recovery after surgery $\left(\operatorname{ERAS}\left({ }^{\circledR}\right)\right)$ Society recommendations. World J Surg 2013; 37: 259-84.

23. Nygren J, Thacker J, Carli F, et al. Guidelines for perioperative care in elective rectal/pelvic surgery: enhanced recovery after surgery (ERAS ${ }^{\circledR}$ ) Society recommendations. World I Surg 2013; 37: 285-305.

24. Lassen K, Coolsen MME, Slim K, et al. Guidelines for perioperative care for pancreaticoduodenectomy: Enhanced Recovery After Surgery (ERAS(R)) Society recommendations. World J Surg 2013; 37: 240-8.

25. King PM, Blazeby JM, Ewings P, Kennedy RH. Detailed evaluation of functional recovery following laparoscopic or open surgery for colorectal cancer within an enhanced recovery programme. Int J Color Dis 2008; 23: 795-800.

Received: 5.04.2018, accepted: 10.06.2018. 


\section{Supplementary material: Questionnaire used in the study \\ Questionnaire for patients}

\section{Part 1. (Before surgery)}

Please indicate how important you feel that each of the following targets is for recovery after the surgery.

1. To be counselled preoperatively by the anaesthetist or surgeon

$\begin{array}{cccccccccc}1 & 2 & 3 & 4 & 5 & 6 & 7 & 8 & 9 & 10 \\ \text { Not important } & & & & & & & & & \\ \text { Very important }\end{array}$

2. To avoid fasting before surgery

$\begin{array}{ccccccccc}1 & 2 & 3 & 4 & 5 & 6 & 7 & 8 & 9 \\ \text { Not important } & & & & & & & & \text { Very important }\end{array}$

3. To be able to drink shortly before the surgery

$\begin{array}{cccccccccc}1 & 2 & 3 & 4 & 5 & 6 & 7 & 8 & 9 & 10 \\ \text { Not important } & & & & & & & & \text { Very important }\end{array}$

4. To avoid bowel cleaning when possible

$\begin{array}{cccccccccc}1 & 2 & 3 & 4 & 5 & 6 & 7 & 8 & 9 & 10 \\ \text { Not important } & & & & & & & & \text { Very important }\end{array}$

5. To be able to reduce the amount of intravenous fluids postoperatively

$\begin{array}{ccccccccc}1 & 2 & 3 & 4 & 5 & 6 & 7 & 8 & 9 \\ \text { Not important } & & & & & & & & \text { Very important }\end{array}$

6. To avoid sedative drugs before surgery

$\begin{array}{ccccccccc}1 & 2 & 3 & 4 & 5 & 6 & 7 & 8 & 9 \\ \text { Not important } & & & & & & & & \text { Very important }\end{array}$

7. To be warmed up in the operative room during surgery

$\begin{array}{cccccccccc}1 & 2 & 3 & 4 & 5 & 6 & 7 & 8 & 9 & 10 \\ \text { Not important } & & & & & & & & \text { Very important }\end{array}$

8. To be treated with minimally invasive techniques

$\begin{array}{cccccccccc}1 & 2 & 3 & 4 & 5 & 6 & 7 & 8 & 9 & 10 \\ \text { Not important } & & & & & & & & & \text { Very important }\end{array}$

9. To be catheterized as briefly as possible

$\begin{array}{cccccccccc}1 & 2 & 3 & 4 & 5 & 6 & 7 & 8 & 9 & 10 \\ \text { Not important } & & & & & & & & & \end{array}$

10. To avoid the use of a nasogastric tube

$\begin{array}{cccccccccc}1 & 2 & 3 & 4 & 5 & 6 & 7 & 8 & 9 & 10 \\ \text { Not important } & & & & & & & & & \end{array}$

11. To avoid the use of abdominal drains

$\begin{array}{cccccccccc}1 & 2 & 3 & 4 & 5 & 6 & 7 & 8 & 9 & 10 \\ \text { Not important } & & & & & & & & & \end{array}$

12. To be completely free of pain

$\begin{array}{ccccccccc}1 & 2 & 3 & 4 & 5 & 6 & 7 & 8 & 9 \\ \text { Not important } & & & & & & & & \end{array}$

13. To get painkillers immediately when needed, on request

$\begin{array}{ccccccccc}1 & 2 & 3 & 4 & 5 & 6 & 7 & 8 & 9 \\ \text { Not important } & & & & & & & & \text { Very important }\end{array}$

14. To be completely free of nausea and vomiting

$\begin{array}{cccccccccc}1 & 2 & 3 & 4 & 5 & 6 & 7 & 8 & 9 & 10 \\ \text { Not important } & & & & & & & & \text { Very important }\end{array}$

15. To be able to move the bowels as soon as possible (no constipation or flatulence)

$\begin{array}{cccccccccc}1 & 2 & 3 & 4 & 5 & 6 & 7 & 8 & 9 & 10 \\ \text { Not important } & & & & & & & & & \text { Very important }\end{array}$

16. To be able to drink and eat as soon as possible after surgery

$\begin{array}{cccccccccc}1 & 2 & 3 & 4 & 5 & 6 & 7 & 8 & 9 & 10 \\ \text { Not important } & & & & & & & & \text { Very important }\end{array}$


17. To be allowed a normal diet at will after the operation

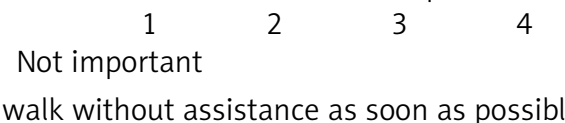

18. To be able to walk without assistance as soon as possible

$\begin{array}{cccccccccc}1 & 2 & 3 & 4 & 5 & 6 & 7 & 8 & 9 & 10 \\ \text { Not important } & & & & & & & & \text { Very important }\end{array}$

19. To be discharged from the hospital as quickly as possible

$\begin{array}{cccccccccc}1 & 2 & 3 & 4 & 5 & 6 & 7 & 8 & 9 & 10 \\ \text { Not important } & & & & & & & & \text { Very important }\end{array}$

20. To be able to return quickly to everyday activities

$\begin{array}{cccccccccc}1 & 2 & 3 & 4 & 5 & 6 & 7 & 8 & 9 & 10 \\ \text { Not important } & & & & & & & & \text { Very important }\end{array}$

21. To be able to contact the doctor when needed

$\begin{array}{cccccccccc}1 & 2 & 3 & 4 & 5 & 6 & 7 & 8 & 9 & 10 \\ \text { Not important } & & & & & & & & \text { Very important }\end{array}$

22. To be checked up by telephone after hospital discharge

$\begin{array}{cccccccccc}1 & 2 & 3 & 4 & 5 & 6 & 7 & 8 & 9 & 10 \\ \text { Not important } & & & & & & & & & \text { Very important }\end{array}$

Part 2. (On discharge)

Please indicate how difficult/important each of the following issues was after the surgery.

23. Abdominal pain, including pain in the wound area

$\begin{array}{cccccccccc}1 & 2 & 3 & 4 & 5 & 6 & 7 & 8 & 9 & 10 \\ \text { Not a problem } & & & & & & & & \text { Major problem }\end{array}$

24. Bowel functions (nausea, vomiting, constipation, diarrhoea, others)

$\begin{array}{cccccccccc}1 & 2 & 3 & 4 & 5 & 6 & 7 & 8 & 9 & 10 \\ \text { Not a problem } & & & & & & & & & \end{array}$

25. Eating and drinking

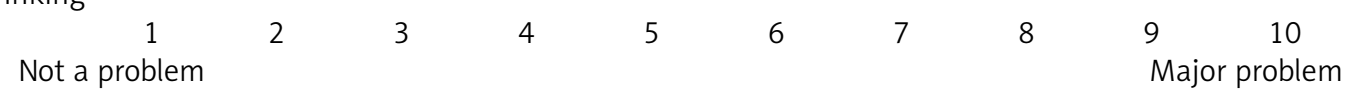

26. Walking without assistance

$\begin{array}{cccccccccc}1 & 2 & 3 & 4 & 5 & 6 & 7 & 8 & 9 & 10 \\ \text { Not a problem } & & & & & & & & & \end{array}$

27. Weakness

$\begin{array}{cccccccccc}1 & 2 & 3 & 4 & 5 & 6 & 7 & 8 & 9 & 10 \\ \text { Not a problem } & & & & & & & & & \text { Major problem }\end{array}$

\section{How important was it for you:}

28. To avoid fasting before surgery

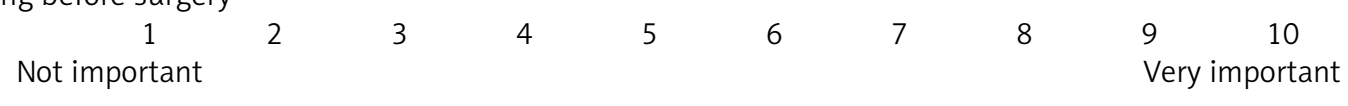

29. To be able to drink shortly before the surgery

$\begin{array}{cccccccccc}1 & 2 & 3 & 4 & 5 & 6 & 7 & 8 & 9 & 10 \\ \text { Not important } & & & & & & & & & \end{array}$

30. To avoid bowel cleaning

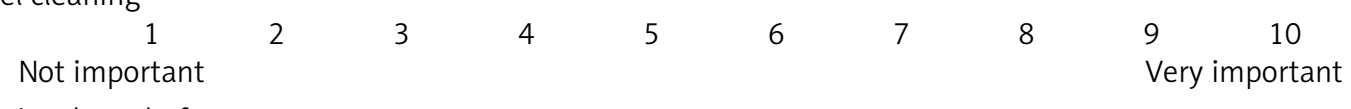

31. To avoid sedative drugs before surgery

$\begin{array}{cccccccccc}1 & 2 & 3 & 4 & 5 & 6 & 7 & 8 & 9 & 10 \\ \text { Not important } & & & & & & & & & \text { Very important }\end{array}$

32. To be warmed up during and after the surgery

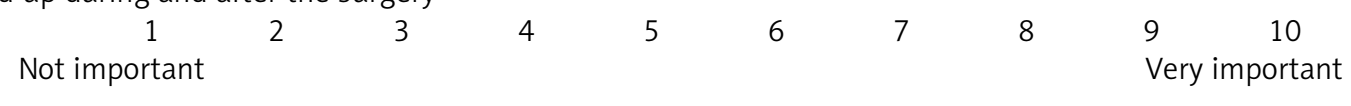


33. To be treated with minimally invasive techniques

$\begin{array}{ccccccccc}1 & 2 & 3 & 4 & 5 & 6 & 7 & 8 & 9 \\ \text { Not important } & & & & & & & & \text { Very important }\end{array}$

34. To be catheterized as briefly as possible or not at all

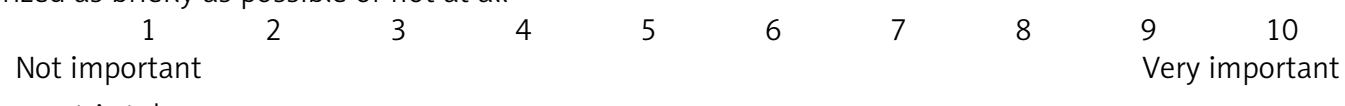

35. To avoid a nasogastric tube

$\begin{array}{cccccccccc}1 & 2 & 3 & 4 & 5 & 6 & 7 & 8 & 9 & 10 \\ \text { Not important } & & & & & & & & \text { Very important }\end{array}$

36. To avoid the use of abdominal drains or to remove them early

$\begin{array}{ccccccccc}1 & 2 & 3 & 4 & 5 & 6 & 7 & 8 & 9 \\ \text { Not important } & & & & & & & & \end{array}$

37. To have the amount of intravenous fluids postoperatively reduced

$\begin{array}{cccccccccc}1 & 2 & 3 & 4 & 5 & 6 & 7 & 8 & 9 & 10 \\ \text { Not important } & & & & & & & & & \text { Very important }\end{array}$

38. To prevent nausea and vomiting

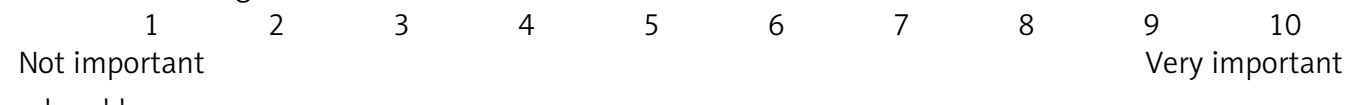

39. To prevent bowel problems

$\begin{array}{ccccccccc}1 & 2 & 3 & 4 & 5 & 6 & 7 & 8 & 9 \\ \text { Not important } & & & & & & & & \end{array}$

40. To be encouraged to eat and drink shortly after surgery

$\begin{array}{cccccccccc}1 & 2 & 3 & 4 & 5 & 6 & 7 & 8 & 9 & 10 \\ \text { Not important } & & & & & & & & & \text { Very important }\end{array}$

41. To be encouraged to walk without assistance shortly after surgery

$\begin{array}{cccccccccc}1 & 2 & 3 & 4 & 5 & 6 & 7 & 8 & 9 & 10 \\ \text { Not important } & & & & & & & & & \text { Very important }\end{array}$

42. To be allowed a normal diet soon after surgery

$\begin{array}{cccccccccc}1 & 2 & 3 & 4 & 5 & 6 & 7 & 8 & 9 & 10 \\ \text { Not important } & & & & & & & & & \text { Very important }\end{array}$

43. To be able to return quickly to everyday activities

$\begin{array}{cccccccccc}1 & 2 & 3 & 4 & 5 & 6 & 7 & 8 & 9 & 10 \\ \text { Not important } & & & & & & & & & \text { Very important }\end{array}$

How would you rate:

44. Being counselled preoperatively by the anaesthetist or surgeon

$\begin{array}{ccccccccccc}1 & 2 & 3 & 4 & 5 & 6 & 7 & 8 & 9 & 10 \\ \text { Very bad } & & & & & & & & & & \text { Very good }\end{array}$

45. Pain management

$\begin{array}{cccccccccc}1 & 2 & 3 & 4 & 5 & 6 & 7 & 8 & 9 & 10 \\ \text { Very bad } & & & & & & & & & \end{array}$

46. Being able to receive pain medication on request

$\begin{array}{ccccccccccc}1 & 2 & 3 & 4 & 5 & 6 & 7 & 8 & 9 & 10 \\ \text { Very bad } & & & & & & & & & \text { Very good }\end{array}$

47. Being able to walk without assistance shortly after surgery

$\begin{array}{cccccccccc}1 & 2 & 3 & 4 & 5 & 6 & 7 & 8 & 9 & 10 \\ \text { Very bad } & & & & & & & & & \end{array}$

48. Early discharge

$\begin{array}{cccccccccc}1 & 2 & 3 & 4 & 5 & 6 & 7 & 8 & 9 & 10 \\ \text { Very bad } & & & & & & & & & \text { Very good }\end{array}$

49. Being allowed to recover at home

$\begin{array}{cccccccccc}1 & 2 & 3 & 4 & 5 & 6 & 7 & 8 & 9 & 10 \\ \text { Very bad } & & & & & & & & & \end{array}$


50. Being able to contact medical staff when needed

$\begin{array}{cccccccccc}1 & 2 & 3 & 4 & 5 & 6 & 7 & 8 & 9 & 10 \\ \text { Very bad } & & & & & & & & & \text { Very good }\end{array}$

51. Being checked up by telephone after hospital discharge

$\begin{array}{cccccccccc}1 & 2 & 3 & 4 & 5 & 6 & 7 & 8 & 9 & 10 \\ \text { Very bad } & & & & & & & & & \end{array}$

Part 3. (telephone survey, 2 weeks after discharge)

Please answer YES or NO to the following questions.

Since the moment of discharge until today, did you:

52. Feel abdominal pain (including the wound area)?

53. Feel fatigued?

54. Have nausea/vomiting/constipation/diarrhoea?

55. Have problems with eating or drinking?

56. Feel afraid about your health due to early hospital discharge?

57. Feel the need for an extended length of stay in hospital?

58. Seek medical help outside our department as a result of postoperative complications?

59. Were you satisfied with this type of perioperative care?

60. Would you recommend use of a similar perioperative care system to other patients? 